\title{
Comparison of ECG T-wave Duration and Morphology Restitution Markers for Sudden Cardiac Death Prediction in Chronic Heart Failure
}

\author{
Julia Ramírez ${ }^{1}$, Michele Orini ${ }^{2}$, Esther Pueyo ${ }^{1}$ Pablo Laguna ${ }^{1}$ \\ ${ }^{1}$ Biomedical Signal Interpretation and Computational Simulation (BSICoS) group, Aragón Institute \\ of Engineering Research (I3A), IIS Aragón, University of Zaragoza, Zaragoza, Spain, and \\ Biomedical Research Networking Center in Bioengineering, Biomaterials and Nanomedicine \\ (CIBER-BBN), Zaragoza, Spain. \\ ${ }^{2}$ Mechanical Engineering Department, University College London, London, UK.
}

\begin{abstract}
An index of T-wave morphology restitution, TMR, has previously shown to be a sudden cardiac death (SCD) predictor in a population of chronic heart failure (CHF) patients. The aim of this study is to compare the predictive value of $T M R$, T-wave width restitution (TWR), $T$ peak-to-end (Tpe) morphology restitution (TpeMR) and Tpe duration restitution (TpeR) indices in the same CHF population. Holter ECG recordings from $651 \mathrm{CHF}$ patients of the MUSIC study, including SCD victims and survivors, were analyzed. TMR was significantly correlated with $T W R(\rho=0.66)$, TpeMR $(\rho=0.70)$ and Tpe $R$ $(\rho=0.42)$. SCD victims showed significantly higher values of TMR,TWR and TpeMR than the rest of patients, with TMR being the index most strongly associated with SCD ( $p=0.002, p=0.006$ and $p=0.011$, respectively). TpeR values were only borderline significantly higher in $S C D$ victims $(p=0.061)$. Univariate Cox analysis showed that $T M R$ was the restitution index with the strongest predictive value (hazard ratio (HR) of 1.466, $p<0.001$ ), followed by TWR (HR of 1.295, $p=0.005$ ), TpeR (HR of 1.297, $p=0.004$ ) and TpeMR (HR of 1.164, $p=0.020$ ). In conclusion, considering the predictive value of the four $T$ wave restitution indices, TMR is the preferred index for $S C D$ risk stratification, followed by TpeMR. However, the marker $T W R$ could also be used for SCD prediction when computational efficiency is an issue.
\end{abstract}

\section{Introduction}

The action potential duration (APD) restitution (APDR) curve represents the interaction between local APD and local cycle length [1]. The morphology of the T-wave reflects the distribution of the repolarization sequence along the ventricle [2-4]. Therefore, the spatio-temporal inhomogeneity of the ventricular repolarization process as a response to changes in heart rate may be captured by an index measuring T-wave morphological changes.

In a recent study, the index of T-wave morphology restitution $(T M R)$ was proposed and proved to predict sudden cardiac death (SCD) in a population of 651 chronic heart failure (CHF) patients [5]. The TMR index is calculated as the difference in the morphology of two T-waves measured at two RR interval (RRI) bins. These bins represent the extremes of the intra-subject RRI range, $\Delta R R I$, as long as each bin contains at least 50 beats. To compute TMR, the morphological T-wave differences are normalized by $\Delta$ RRI (see Figure 1). Although there are studies reporting that the T-peak-T-end (Tpe) interval has stronger predictive value for arrhythmic risk than the overall T-wave width (TW) [6], the comparison of the predictive value of their respective restitution indices, TpeR and $T W R$ has never been performed. The comparison of the predictive value of the restitution of the overall T-wave morphology $(T M R)$ and the restitution of the T-peak-to-end (Tpe) morphology $(T p e M R)$ has also never been assessed before. The results of these comparisons may indicate new methods for optimizing the predictive value of ECG repolarization markers and improve their mechanistic interpretation.

In this study we calculated the SCD predictive value of several repolarization ECG markers, including restitution of T-wave morphology and duration indices, in a cohort of $651 \mathrm{CHF}$ patients.

\section{Materials and Methods}

\subsection{Materials}

The study population included 651 consecutive patients with symptomatic CHF corresponding to NYHA classes II and III enrolled in the MUSIC study, a prospective, multicenter study designed to assess risk predictors for cardiovascular mortality in ambulatory patients with CHF [7]. A 
two- or three-lead 24-hour Holter ECG sampled at $200 \mathrm{~Hz}$ was recorded in each patient at enrolment using ELA Medical equipment (Sorin Group, Paris, France). Baseline demographic and clinical data in sinus rhythm were available for the analysis. The MUSIC study included patients with both reduced and preserved LVEF, ranging from $10 \%$ to $70 \%$. The study protocol was approved by the institutional investigation committees and all patients signed informed consent. No medications were withdrawn during Holter monitoring.

Patients were followed up every 6 months for a median of 48 months. SCD was defined as (1) a witnessed death occurring within 60 minutes from the onset of new symptoms unless a cause other than cardiac failure was obvious, (2) an unwitnessed death $(<24$ hours) in the absence of preexisting progressive circulatory failure or other causes of death, or (3) death during attempted resuscitation. End points were reviewed and classified by the MUSIC Study Endpoint Committee.

\subsection{ECG Pre-processing}

Preprocessing of the ECG signals was performed using custom-written software and included low pass filtering at $40 \mathrm{~Hz}$ to remove electric and muscle noise, cubic splines interpolation for baseline wander removal, and ectopic beats detection.

Principal Component Analysis (PCA) was applied over the two-or-three available ECG leads to emphasize the energy of the T-wave and improve its delineation [8]. The PCA training matrix was built by only considering the samples from the T-waves on each lead. First, a singlelead delineation technique was applied to select the samples from the T-wave and compute the PCA training matrix. Then, the first principal component was computed and delineated using a single-lead technique [9]. From the delineation marks, the RR interval series was obtained and the $\mathrm{T}$-waves were selected using the obtained delimitation marks.

\subsection{T-wave Restitution Indices}

First, the histogram of the RR series was calculated during the entire 24-h recording, and it was divided into 10ms-wide bins. Then, only the bins having at least 50 occurrences were selected. Next, two RR bins, distributed symmetrically around the median RR, were chosen as those defining the extremes of the intra-subject $R R$ range, $\Delta R R$, for the study. Then, the T-waves corresponding to the beats associated with the RR intervals within these two bins were considered for the analysis (Figure 1).

A methodology described in a previous publication [10] was applied to calculate the mean warped T-waves of those $\mathrm{T}$-waves selected from each RR bin. These mean warped

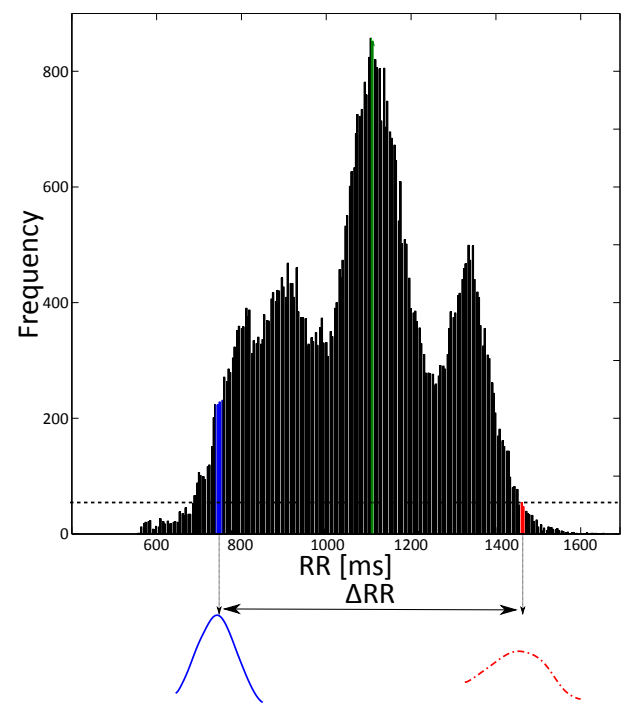

Figure 1. Histogram of RR divided into 10-ms wide bins. The pair of most distant bins, symmetrically distributed around the median and constrained to contain a minimum of 50 beats, are selected, defining the extreme intra-subject $\mathrm{RR}$ range, $\Delta \mathrm{RR}$. Two mean warped T-waves, each of them representative of the T-wave morphology in each bin, are subsequently derived.

$\mathrm{T}$-waves are representative of the average T-wave morphology at each RR. In addition, the morphology of the T-wave is highly dependent on the history of previous RR intervals [11], which means that the morphology of the T-wave coming from a history of previous RR interval values longer than the current one is different than that corresponding to a T-wave coming from a history of previous RR interval values shorter than the current one. Due to the fact that the mean warped T-wave is not just a simple signal averaging, but it rather stretches (warps) the temporal domains of the T-waves [10], it compensates for the morphological differences due to the different histories of RR interval values [12].

\subsubsection{Overall T-wave Restitution Indices}

- T-wave Morphology Restitution: The gravity centres of both mean-warped T-waves were aligned to make the optimal warping function, $\gamma^{*}$, only dependent on changes in the T-wave morphology, and not on global shifts. The average difference, in ms, between the temporal domain of the mean warped T-waves is quantified by $d_{w}^{\mathrm{TW}}$ [10], and measures the morphological differences in the time domain between the two mean warped T-waves. The super script $T W$ indicates that the overall $\mathrm{T}$-wave is considered. The index $T M R$ was calculated by dividing $d_{w}^{\mathrm{TW}}$ by $\Delta R R$. Therefore, $T M R$ is a measure of the T-wave morphological change per RR increment (Figure 2). 
- T-wave Width Restitution: The index $T W R$ was calculated by dividing the difference between each mean warped T-wave width, $T W_{1}$ and $T W_{2}$, by $\Delta R R$. Therefore, $T W R$ is a measure of the T-wave width change per $\mathrm{RR}$ increment (Figure 2). Note that no warping between the two T-waves is required to calculate $T W R$.

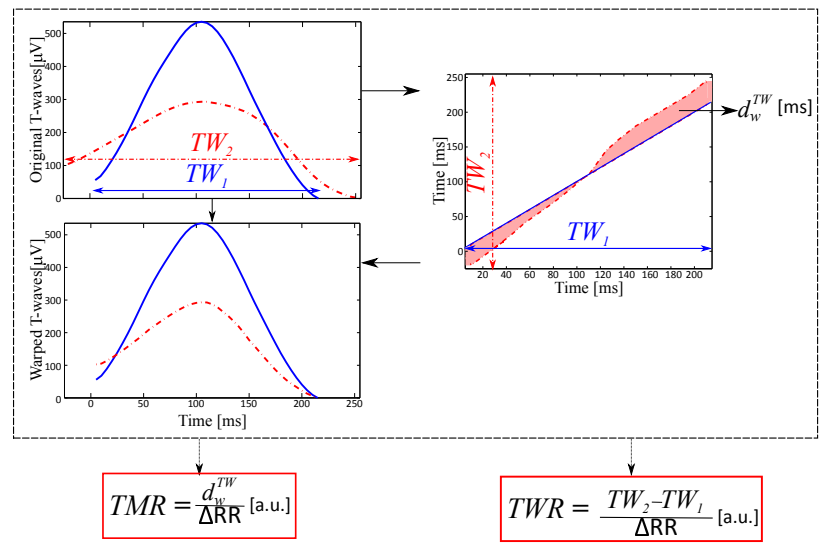

Figure 2. Calculation of the index of T-wave morphology restitution, $T M R$, and of the index of T-wave width restitution, $T W R$.

\subsubsection{T-peak-to-end Restitution Indices}

- T-peak-to-end Morphology Restitution: The peak and end marks from the mean warped T-waves were delineated using a single-lead delineation method [9]. Only the morphology of the mean warped T-waves from the peak to the end marks was considered. The warping was applied by comparing both T-peak-to-end mean warped T-waves [10], and their morphological variability was subsequently quantified by $d_{w}^{\mathrm{Tpe}}$, where the superscript $T p e$ indicates that only the T-peak-to-end morphology is being considered. Finally, the index TpeMR was calculated by dividing $d_{w}^{\mathrm{Tpe}}$ by $\Delta R R$. Therefore, $T p e R^{\mathrm{Tw}}$ is a measure of the T-peakto-end morphological change per RR increment (Figure 3). - T-peak-to-end Interval Restitution: The index TpeR was calculated by dividing the difference between each Tpe interval, $T p e_{1}$ and $T p e_{2}$, by $\Delta R R$. Therefore, $T p e R$ is a measure of the Tpe change per RR increment (Figure $3)$. Note that no warping between the two T-waves is required to calculate TpeR.

\subsection{Statistical Analyses}

The two-tailed Mann-Whitney test was used to evaluate the association of TMR,TWR, TpeR and TpeMR with SCD. Correlation was evaluated with the Spearman correlation coefficient. Univariate Cox regression analyses were performed to determine the predictive value of the indices. A value of $\mathrm{p}<0.05$ was considered statistically sig-

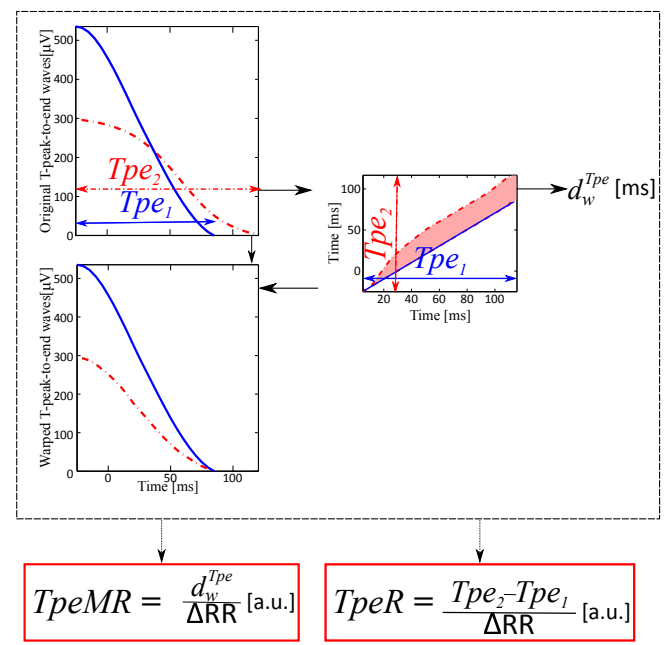

Figure 3. Calculation of the index of T-peak-to-end morphology restitution, TpeMR, and of the index of T-peakto-end restitution, TpeR.

nificant. Statistical analyses were performed using SPSS version 22.0 (SPSS Inc, Chicago, IL).

\section{Results and Discussion}

Table 1 (left panel) shows the Spearman correlation coefficients between each pair of restitution indices. As it can be observed, a moderate correlation was found between the two restitution markers based on morphology, TMR and TpeMR, suggesting that the information contained in Tpe $M R$ is influencing the one expressed by $T M R$. The highest correlation coefficient was found between $T M R$ and $T W R$, which reflects the restitution of the T-wave width, suggesting that the variations in $T M R$ as a response to heart rate are influenced by the T-wave width. There was also a moderate correlation between $T W R$ and TpeR, the two interval-based restitution indices. Finally, only a weak correlation between $T W R$ and Tpe $M R$ was observed, possibly reflecting their differences in both intervals of analysis and nature of the indices.

Table 1 (middle panel) shows the median (interquartile range) of the four $\mathrm{T}$-wave restitution indices in the group formed by SCD victims and in the group formed by non-SCD victims. The T-wave restitution index most strongly associated with SCD risk was $T M R$, followed by $T W R$ and $T p e M R$. TpeR, on the contrary, only showed borderline significant association with increased SCD risk.

Table 1 (right panel) shows the results from the Univariable Cox regression analyses, demonstrating that the four T-wave restitution indices are associated with SCD risk along the follow-up period. The index $T M R$ showed the highest association with SCD along time. The reason 


\begin{tabular}{|c|c|c|c|c|c|c|c|c|c|c|}
\hline \multicolumn{5}{|c|}{ Correlation } & \multicolumn{4}{|c|}{ Association with SCD } & \multicolumn{2}{|c|}{ Prediction of SCD } \\
\hline & $T M R$ & $T W R$ & TpeMR & TpeR & & SCD victims & non-SCD victims & p-value & HR (IQR) & p-value \\
\hline$T M R$ & - & 0.63 & 0.70 & 0.36 & $T M R$ & $0.048(0.03)$ & $0.041(0.02)$ & 0.002 & $1.466(1.235-1.741)$ & $<0.001$ \\
\hline$T W R$ & - & - & 0.36 & 0.62 & $T W R$ & $0.233(0.09)$ & $0.203(0.13)$ & 0.004 & $1.350(1.112-1.638)$ & 0.002 \\
\hline Tpe $M R$ & - & - & - & 0.47 & Tpe $M R$ & $0.043(0.05)$ & $0.033(0.02)$ & 0.011 & $1.164(1.024-1.323)$ & 0.020 \\
\hline TpeR & - & - & - & - & TpeR & $0.098(0.08)$ & $0.088(0.07)$ & 0.057 & $1.295(1.033-1.624)$ & 0.025 \\
\hline
\end{tabular}

Table 1. Left: Spearman's correlation coefficients. Middle: Median (interquartile range) for the group of SCD victims and the group formed by the rest of patients and Mann-Whitney test p-values between both groups. Right: Hazard ratios (interquartile ranges) and p-value for the Univariate Cox regression analysis. Statistical significance is indicated in bold.

why $T M R$ presents higher significance than $T W R$ can to some extent be attributed to the fact that uncertainties in delineation to compute time intervals are largely affecting robustness of interval indices, while in the case of $T M R$ the results are largely insensitive to delineation errors [10].

\section{Conclusions}

Considering the predictive value of the four T-wave restitution indices, $T M R$ is the preferred index for SCD risk stratification, followed by TpeMR. However, the marker $T W R$ could also be used for SCD prediction when computational efficiency is an issue, based on its correlation with $T M R$. TpeR was the weakest restitution index for SCD prediction in CHF patients.

\section{Acknowledgements}

This work was supported in part by projects TIN201341998-R and DPI2016-75458-R from Spanish Ministry of Economy and Competitiveness (MINECO), Spain, by Aragón Government, Spain and from European Social Fund (EU) through BSICoS group and by the European Research Council (ERC) through project ERC-2014-StG 638284. The computation was performed at the High Performance computing platform of the NANBIOSIS ICTS, CIBER-BBN and I3A, Zaragoza, Spain.

\section{References}

[1] Orini M., Taggart P., Srinivasan N., Hayward M. and Lambiase P.D., "Interactions between activation and repolarization restitution properties in the intact human heart: In vivo whole-heart data and mathematical description," Plos One, vol. 11, no. 9, p. e0161765, 2016.

[2] Burgess M. J., "Relation of ventricular repolarization to electrocardiographic T wave-form and arrhythmia vulnerability," Am J Physiol, vol. 236, no. 3, pp. H391-H402, 1979.

[3] Shimizu W. and Antzelevitch C., "Cellular basis for long QT, transmural dispersion of repolarization, and torsade de pointes in the long QT syndrome," J Electrocardiol, vol. 32, no. Suppl, pp. 177-184, 1999.
[4] Gima K. and Rudy Y., "Ionic current basis of electrocardiographic waveforms: a model study," Circ Res, vol. 90, no. 8, pp. 889-896, 2002.

[5] Ramírez J., Orini M., Mincholé A., Monasterio V., Cygankiewicz I., De Luna A. B., et. al., "T-wave morphology restitution predicts sudden cardiac death in patients with chronic heart failure," J Am Heart Assoc, vol. 6, no. 5, p. e005310, 2017.

[6] M. Yamaguchi et. al., "T wave peak-to-end interval and QT dispersion in acquired long QT syndrome: a new index for arrhythmogenicity.," Clinical Science, vol. 105, no. 6, pp. 671-676, 2003.

[7] Vázquez R., Bayés-Genís A., Cygankiewicz I., PascualFigal D., Grigorian-Shamagian L., Pavon R., et. al., "The MUSIC Risk score: a simple method for predicting mortality in ambulatory patients with chronic heart failure.," $E u$ ropean Heart Journal, vol. 30, pp. 1088-1096, 2009.

[8] Ramírez J., Mincholé A., Laguna P. and Pueyo E., "Characterization of cardiac repolarization response to heart rate changes provoked by a Tilt test," in Computing in Cardiology, vol. 39, pp. 673-676, 2012.

[9] Martínez J.P., Almeida R., Olmos S., Rocha A.P. and Laguna P., "A wavelet-based ECG delineator: evaluation on standard databases," IEEE Transactions on Biomedical Engineering, vol. 51, pp. 570-581, 2004.

[10] Ramírez J., Orini M., Tucker J.D., Pueyo E. and Laguna P., "Variability of ventricular repolarization dispersion quantified by time-warping the morphology of the T-waves.," IEEE Trans Biomed Eng, vol. 64, no. 7, pp. 1619-1630, 2017.

[11] E. Pueyo et. al., "Characterization of QT interval adaptation to RR interval changes and its use as a risk-stratifier of arrhythmic mortality in amiodarone-treated survivors of acute myocardial infarction," IEEE Transactions on Biomedical Engineering, vol. 51, no. 9, pp. 1511-1520, 2004.

[12] Ramírez J., Orini M., Pueyo E., Laguna P., "Quantification of T-wave morphological variability using time-warping methods," EMBEC-NBC, vol. 1, pp. 478-481, 2017.

Address for correspondence:

Julia Ramírez, Campus Río Ebro, I+D Building, D-5.01.1B, c $\backslash$ Mariano Esquillor, s/n, 50018 Zaragoza (Spain),

Julia.Ramirez@unizar.es 